Das Oedema cutis proprium, das ich als Krankheit sui generis betrachten möchte, kann sowohl akut nach Durchnässung, Erkältung, Infektionskrankheiten als nachweisbare Ursache auftreten, es kann aber auch alcut oder allmählich zur vollen Höhe sich entwickeln, ohne daß eine äußere oder innere Schädigung nachzuweisen ist. Die Krankheit kann besonders bei falscher Diagnose sich jahrelang hinziehen, ohne daß jemals etwas anderes als ein mehr oder weniger mächtiges Ödem der Haut sich entwickelt, während es bei zweckentsprechender Behandlung nach kürzerer oder längerer Frist meist in Heilung übergeht. EPPINGer hat zwar auch tödlichen Ausgang an Marasmus in einzelnen Fällen beobachtet.

Wie verhält sich nun das Oedema cutis proprium zu denjenigen Odemen, die gleichzeitig mit Erkerankung der Nieren auftreten und sowohl bezüglich ihrer Lokalisation in der Haut, als auch gegenüber der Kochsalzentziehung ein völlig analoges Verhalten zeigen? Sind sie, wie Lichтwiтz annimmt, parallel verlaufende Erkrankungen oder nach VolHaRd die Folge nephrogener Gifte, die aus der degenerativen Metamorphose der Nierenepithelien in die Blutbahn gelangen?

Gegen die Ansicht VolHARDs spricht die auch von EPPINGER häufig konstatierte Tatsache, daß Heilung, ja Dauerheilung des Hautödems, auch bei Nephritis, sei es nach Kochsalzentziehung sei es nach Schilddrüsentherapie, eintreten kann unter gleichzeitiger Verschlimmerung der Nierenerkrankung. Es hat sogar den Anschein, als ob durch die akute Mobilisierung der Chlordepots und ihre Ausscheidung durch die Nieren der renale Degenerationsprozeß neu angefacht werden könnte.

Wir müssen deshalb mit LIcHTwITz annehmen, daß Haut und Nierenschädigung parallel laufen und derselben Giftwirkung ihre Entstehung verdanken. Manche klinische Beobachtungen sprechen sogar dafür, daß die Houterkrankung der Nierenschädigung vorausgehen kann. Ein klassisches Beispiel dafür ist der Hydrops scarlatinosus, sine nephritide, ein typisches, durch infektiöse Hautschädigung bedingtes Oedema cutis proprium, das bald für sich allein bestehen bleibt und abheilt, aber auch nicht selten wenige Tage nach seinem Auftreten von einer Nephritis begleitet ist. Auch EPPINGER beschreibt einen Fall von schwersiem Hautödem nach Durchnässung, indem die nephritischen Symptome sich erst einstellten, als die Hautwassersucht durch Schilddrüsendarreichung beseitigt war.

Trennen wir alle diejenigen Odeme bei Nierenerkrankung, die auf zentrale oder periphere Gefäßschädigung zurückgeführt werden müssen, streng vom eigentlichen Hautödem, wie es in seiner Mächtigkeit sich gerade bei der Nephrose entwickelt, so drängt sich uns die Hypothese auf, ob nicht gerade durch die Miterkrankung der Haut der meist günstige Verlauf dieser in ihren Symptomen oft so bedrohlich erscheinenden Nierenerkrankung bedingt wird, ob nicht auch hier die Haut als das Schutzorgan des gesamten Organismus auftritt, das die Funktion besitzt, die lebenswichtigen Organe, in diesem Falle die Nieren, vor schweren Schädigungen zu bewahren. Wenn wir für Haut- und Nierenerkrankung dasselbe Gift verantwortlich machen, so kann durch Giftbindung in der Haut eine Nierenerkrankung verhütet oder doch zum wenigsten in ihrem Verlaufe gemildert werden. Dafür sprechen auch die Erfahrungen EPPINGERs, der nach Beseitigung des Hautödems Verschlimmerung des Nierenleidens konstatieren mußte, and die Strausssche Beobachtang, die auch von anderen Autoren bestätigt wird, daß das Hautödem einen Schutz gegen Urämie gewähren und daß sein $z u$ schnelles Schwinden den Ausbruch der Urämie begünstigen kann. Beide Tatsachen aber legen die Vermutung nahe, da $B$ in den Hautödemen nicht nur Kochsalz und Wasser, sondern auch nierenschädigende Gifte gebunden sind.

\section{KURZE BEMERKUNGEN ZUR KLINIK DER GALLENSTEINERKRANKUNGEN.}

\section{Von}

Prof. Dr. Lothar Heidenhain.

Direktor der Chirurg. Abteilung des städt. Krankenhauses za Worms.

In dieser Zeitschrift (Nr. 2I, S. I078) empfahl vor kurzem Herr Pfeffer Tabletten von Desoxycholsäure allein oder in Irombination mit kleinen Mengen Pfefferminzöl (Degalol) als ein wertvolles Mittel zur Bekämpfung der Gallensteine, und: dies auf Grund klinischer Beobachtung von I 4 Fällen. Wenn. von hochgeschätzter interner Seite, hier der medizinischen Klinik der Düsseldorfer Akademie, dem Praktiker derartige Empfehlungen gegeben werden, so ist es für den Chirurgen Pflicht, sich seinerseits dazu zu äußern, seinen Standpunkt. ebenfalls $\mathrm{zu}$ vertreten.

Zunächst seien, um den Leser ins Bild zu bringen, die Hauptpunkte von Herrn PFEFFers Mitteilungen wiedergegeben. Ich lasse ihn selbst reden: „Um die Cholelithiasis wirksam zu bekämpfen, handelt es sich für den inneren Mediziner darum, erstens die Bildung der Gallensteine zu verhindern, zweitens die vorhandenen Gallensteine aufzulösen oder zum Abgang zu bringen." ,Die Auflösung der gebildeten Steine in der Gallenblase ist verschiedentlich beobachtet worden. Sie ist durch Umspülung mit Galle, die reichlich Gallensäure enthält, möglich. Auch hier würde also eine $Z$ ufuhr von Desoxycholsäure fördernd einwirken." "Wir haben, nachdem. wir das Mittel einige Tage hindurch gegeben hatten, eine: bedeutende Abschwächung und meistens ein Aufhören der Gallensteinanfälle gesehen. Der Ikterus und die Leberschwellungen nahmen in allen Fällen prompt ab." "Im Degalol ist also eine wertvolle Erweiterung der Mittel zur Bekämpfung der Gallensteine zu sehen. Es vereinigt in sich die gallentreibende und steinlösende Wirkung."

Hierzu ist zunächst $\mathrm{zu}$ bemerken, daß nach klinischer Beobachtung wie anatomischer Untersuchung . und vieltausendfältiger Erfahrung am Operationstisch, bei Operationen im akuten Anfall wie im Intervall, der sogenannte Gallensteinanfall nicht durch die Steine als solche, also etwa durch ein Wandern der Steine bedingt ist, sondern durch eine akuteoder rezidivierende, leichtere oder schwerere Entzündung der Gallenblase oder der tiefen Gallenwege, oder beider zusammen, also Cholecystitis und Cholangitis. Die Wanderung der Steine aus der Gallenblase in die tiefen Gallenwege ist nur ein sekundäres, wenn auch schwerwiegendes Ereignis. Der Kernpunkt der Diskussion der Gallensteinfragen sind nicht die Steine, trotz der besonderen Rolle, welche sie spielen, sondern es ist die Infektion der Gallenwege. Sie ist es, welche den ersten Anfall und die Rezidive der Anfälle hervorruft, welche die Kranken in zahllosen Fällen schließlich siech macht und tötet.

Zum zweiten wäre zu sagen, daß Auflösung der gebildeten. Steine in der Gallenblase noch nie beobachtet worden ist, denn solche Beobachtung ist unmöglich. Sollte erwidert werden, daß nach schweren ,Gallensteinanfällen" bei Operation oder Sektion die Gallenwege steinfrei befunden worden seien, so. wäre zu entgegnen, daß Cholecystitis sine calculo durchaus nicht selten ist, daB solche Erkrankung klinisch von Cholecystitis calculosa zu unterscheiden unmöglich ist, daß eben in solchem Falle vermeintlicher Steinauflösung die Diagnose „Gallensteinanfall" falsch war. Auflösung der Gallensteine ist schon darum unmöglich, weil bei weitem die meisten von ihnen reichlich unlösliche Kalksalze enthalten. Reine Cholesterinsteine sind recht selten.

Zum weiteren: Daß auf Anwendung irgendeines internen. Mittels nach einigen Tagen die Gallensteinanfälle sich bedeutend abschwächen, ja meistens aufhören, sagt nichts über die Wirksamkeit dieses Mittels. Es ist eben Eigenheit der Gallensteinanfälle, selbst schwerer, daß sie durchschnittlich nach einigen Tagen und, wenn sie recht hartnäckig. sind, nach ein bis zwei Wochen abflauen und vorläufige Genesungeintritt - bis zum Rezidive. Auch über diesen Punkt dürfte allgemeines Einverständnis bestehen. Unsere eigne Erfahrung hierüber ist auch nicht ganz gering, da ich während der ersten I6 Jahre hiesiger Tätigkeit die innere Abteilung des Krankenhauses ebenfalls geleitet, aus dieser Abteilung fast mein ganzes Material betreffend die sogenannten Grenzgebiete der Medizin und Chirurgie herausgezogen habe, die letzten 25 Jahre der Entwicklung der Gallensteinchirurgie handelnd miterlebt und in den ersten Jahren durchaus nicht auf dem. Standpunkt gestanden - den ich übrigens auch jetzt noch nicht vertrete - daß jeder Fall von Gallensteinerkrankung operiert werden soll oder muß. So aber, wie bisher, darf es nicht weiter gehen. In unserem Material an operierten Kranken, 
bis Ende März I920 570 Fälle (bis 3I. März 1922 gestiegen auf 673 Fälle), waren $52,7 \%$ der Kranken verschleppte Fälle, kamen mit ausgesprochener Sepsis oder Peritonitis, Steinen in den tiefen Grallenwegen, Cholangitis septica, Inanition, Myodegeneratio cordis und anderen schweren Komplikationen, oder mit Gallenblasenkrebs in unsere Hände. Die Zahl der Fälle von Krebs auf Basis eines Gallensteinleidens betrug 37 oder $61 / 2 \%$ aller Operierten. In L. ReHNs Material betrug die Zahl der verschleppten Fälle $38,24 \%$ des Zuganges. Gleichsinnige Erfahrungen haben alle anderen Chirurgen gemacht. Bei rechtzeitiger Operation, das ist vor Eintritt lebensgefährdender Komplikationen, beträgt bei uns die Sterblichkeit $\mathrm{I}, 5 \%$, bei schweren Komplikationen aber $\mathrm{I}_{4}$ bis $I_{5} \%$; ein Kranker mit Gallenblasenkrebs ist nach unserer Erfahrung nicht mehr zu retten. Wir haben keinen gesund bleiben sehen. Dabei beträgt die unnittelbare Mortalität der Operationen bei Krebs 30\%. Betrachtet man unsere 4 Todesfälle nach 269 Operationen wegen unkomplizierter Gallenblasenerkrankung, so zeigt sich, daB auch hier noch Allgemeinstörungen, Tuberkulose, schwere Syphilis, Inanition drei Viertel der 4 Todesfälle bilden, so daß man - und dies gilt für die durchschnittlichen Erfahrungen unserer deutschen Kliniken - sagen kann, daß die Operation bei Gallensteinleiden so gut wie ungefährlich ist, wenn operiert wird, ehe lebensgefährdende Komplikationen eingetreten sind. Zahlreiche Statistiken in KEHRS letztem Werk.

Da ich hier doch nur eine Skizze geben kann, so verweise ich auf eine Darstellung der Pathogenese und der Operationsanzeigen bei Gallensteinleiđen, welche ich kürzlich gegeben habe (Naturforscherversammlung Nauheim I920, Münch. med. Wochenschr. I92I, Nr. 39, S. I239). Ich weiß mich in diesen Fragen völlig einig mit ihren besten Kennern unter den Chirurgen, Körte, KüMmell, PopPert, Rehn u. a. Man fragt sich vergeblich, warum eine medizinische Klinik Kranke mit Leberschwellung und Ikterus, bei welchen sie ein Gallensteinleiden annimmt, mit angeblich galletreibenden und steinlösenden Mitteln behandelt, statt sie ungesäumt der chirurgischen Klinik zu überweisen. Operation solcher Kranker, womöglich im ersten Anfalle und in den allerersten Tagen des Anfalles ist dringlich. Bei allen früh Operierten kann man dafür gutsagen, daß sie gesund werden, während man bei Operationen im $\mathrm{x}$-ten Anfalle nach jahrelangem Verlauf weder für den glücklichen Ablauf der Operation - der Komplikation mit Sepsis, Myodegeneratio cordis, Inanition usw. halber - noch für dauernde Heilung unbedingt einstehen kann wegen Steinbildung hoch oben in der Leber oder dauernder: Neigung za cholangitischen Rezidiven. Das Unglück der Kranken ist, daß trotz schwerer und immer wieder rezidivierender Anfälle über Jahre und Jahrzehnte mit ,,inneren Kuren“" gearbeitet wird, statt sich selbst klarzumachen, da B in solchen und vielen frischeren Fällen, auf welche ich hier nicht eingehen kann, irreparabele Veränderungen vorliegen, bei welchen nur das Messer helfen kann, und den Kranken den Entschluß zur operation dadurch zu erleichtern, daß man ihnen klarmacht, daß das Leiden selbst wesentlich gefährlicher für Gesundheit und Leben sei als die Operation. Denn so liegen die Verhältnisse. Mit Mühe haben wir durch andauernde Deinonstrationen am Operationstische erreicht, daß uns die Hausärzte die Gallensteinkranken rechtzeitiger wie früher, oft mit sehr guten Diagnosen und Indikationsstellungen zusenden. Kommen nun wieder interne Kliniker mit, ,galletreibenden und steinlösenden" Mitteln, für deren Wirksamkeit jeder Beweis fehlt, so wird verdorben, was wir gewonnen haben, die Verringerung der Sterblichkeit an Gallensteinleiden. Locker lassen wir nicht. Ich verweise auf den Aufsatz von ZöPFEL (Klin. Wochenschr. 1922, Nr. 12, S. 583), welcher den Vergleich mit den Wurmfortsatzentzündungen, den ich in Nauheim schon heranzog, vortrefflich weiter ausführt. Im Kampfe um die Wurmfortsatzerkrankungen haben wir erreicht, da $\beta$ die Einsicht in die Notwendigkeit rechtzeitiger Operation allgemein geworden ist. In der Frage der Gallensteinleiden müssen und werden wir es auch erreichen. Hier in unserem Kreise sind wir auf dem besten Wege dazu. Daß jede "Blinddarmentzündung" unbedingt und so schnell wie möglich operiert werden müsse, finden hier Ärzte wie Kranke völlig selbstverständiich. Das Ergebnis in diesem Falle ist, daß wir von 1897 bis 1922 , also in 2.5 Jahren, auf 2303 Operationen wegen akuter Epityphlitis - und wir operieren seit 1900 unbedingt in jedem Falle, gleichviel an welchem Tage der Erkrankung er eingeliefert wird I Io oder $4,78 \%$ Todesfälle haben. Lassen wir alle die relativ einfacheren Fälle, also die gewöhnlich akute Epityphlitis mit und ohne Perforation, mit Frühexsudat oder einigen EBlöffeln Eiter in der Umgebung des Wurms, mit oder ohne Gangrän des Wurms, mit großen und kleinen, frischen und alten Abscessen, beiseite und beziehen die Todesfälle allein auf die $7^{06}$ Fälle freier eiteriger und jauchiger Peritonitis, welche sich unter obigen 2303 Fällen befanden, so ergibt auch

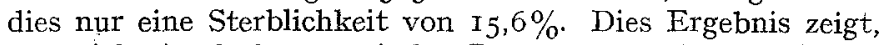
was sich durch konzentrische Zusammenarbeit von Ärzteschaft und Klinik erreichen läßt. Im Gebiete der Gallenstein. erkrankungen ist bei uns eine sehr wesentliche Besserung ganz unverkennbar, Abnahme der verbummelten Fälle, der siechen Kranken, der Fälle schwerer Sepsis usw., Zunahme der Operationen wegen akuter Cholecystitis, wegen akuter Empyeme, vor allem wegen Ikterus mit Leberschwellung, hinter welchem Bilde sich, handelt es sich überhaupt um Gallensteine, zumeist die Fälle von chronischen - sit venia verbo - Choledochussteinen verbergen, welche ohne Operation alle dem Siechtum verfallen sind. Es kann Jahre leidlichen, ja zeitweise guten Befindens dauern: das eine ist und bleibt sicher, daß der Träger eines Choledochussteines an diesem zugrunde geht, wenn nicht Operation helfend eintritt. Die Operation aber im akuten Anfall, womöglich im ersten, ist leicht, denn der Choledochus ist weit; die tiefen Gallenwege sicher steinfrei zu machen, gelingt mühelos. Die Genesung von der Operation geht glatt und schnell. Jetzt nach der Operation treten die Methoden der internen Medizin ein. Schon in den ersten Tagen beginnt eine ausgiebige Trinkkur, um die Infektionserreger aus den Gallenwegen auszuschwemmen. Eine gewisse Neigung zu leichten cholangitischen Rezidiven, bedingt entweder durch Wiederaufflackern der nicht ganz ausgetilgten Infektion der tiefen Gallenwege oder durch Neuinfektion vom Darmkanal aus, besteht bei manchen Kranken nach der Operation fort, führt hier und da zu leichteren Schmerzanfällen. Zweimal im Jahre auf einige Wochen soll die Trinkkur in den ersten Jahren wiederholt werden, gleichviel ob Beschwerden bestanden oder nicht. Soweit ich sehe, tritt auf die Dauer fast immer völlige Gesundung ein. Die Neigung zu Rückfällen, ,des Schnupfens der tiefen Gallenwege", wie ich es den Kranken gegenüber nenne, verliert sich. Auch die Zahl der cholangitischen Rezidive würde sich verringern, im Idealfalle fast zum Verschwinden bringen lassen, wenn immer rechtzeitig operiert würde. Denn die Neigung $z u$ cholangitischen Rezidiven zeigt sich vorzugsweise bei den Kranken, welche im Laufe von Jahren viele, oft ungezählte Anfälle von Ikterus mit Leberschwellung gehabt haben.

\section{BESCHÄFTIGUNGSNEURITIS DER GLAS- SCHLEIFER.}

Von

Landesgewerbearzt Dr. TELEKy, Düsseldorf.

In mehrfacher Art kann Arbeit und Beruf zur Entstehung von Neuritis führen: durch Giftwirkung, toxische Neuritis, als bekanntestes Beispiel die Bleilähmung; durch Überanstrengung, durch Druck auf Muskeln und die in ihnen vorhandenen Nervenendigungen, durch Druck auf den Nerv in seinem Verlaufe. Häufig wirken zwei dieser Ursachen zusammen oder ist wenigstens ihr Zusammenwirken bestimmend für die Lokalisation der Erkrankung. So befällt die Bleilähmung die am stärksten angestrengten Muskelgruppen, wie - neben anderen - auch ich durch genaue Darlegung der Arbeitsverhältnisse bei 28 Fällen von Bleilähmung eigener Beobachtung nachzuweisen mich bemühte (TELEKY, Zur Kasuistik der Bleilähmung. Ein Beitrag zur Edingerschen Aufbrauchtheorie. Dtsch. Zeitschr. $f$. Nervenheilk. 37, 234. I909) und wofür wohl das bekannteste Beispiel die Bleilähmung der 